\title{
Fiche pratique
}

\section{Comment optimiser une présentation assistée par ordinateur?}

\author{
Tristan BOURCIER, Laurent LAROCHE \\ Centre H ospitalier N ational d'O phtalmologie des Q uinze-Vingts, \\ Faculté de médecine Saint-Antoine- U niversité Paris 6 - Paris
}

\section{Définition}

Le contenu d'une présentation assistée par ordinateur (PAO) est plus important que son style. Toutefois, il existe un certain nombre de règles permettant d'améliorer la mise en forme d'une PAO .

\section{D isposition générale de la diapositive}

- Utiliser le mode paysage car les diapositives peuvent contenir plus d'informations quand elles ont orientées horizontalement.

- Utiliser des grilles et des masques communs à toutes les diapositives du fichier afin d'uniformiser la présentation.

\section{Titre}

- Utiliser des titres simples et un titre pour chaque diapositive afin de donner la clé au contenu de la diapositive tout en ne reproduisant pas l'information contenue dans le texte.

\section{Textes}

- U tiliser la « règle des 6 par 6 »: pas plus de 6 mots par ligne de texte, pas plus de 6 lignes par diapositives. II est donc important d'utiliser des puces et mots clés faciles à lire, sans faire de phrases complètes sauf si elles sont très courtes.

- L'alignement du texte à gauche, sans justifier la marge à droite, est plus facileà lire.

\section{Puces}

- Les puces ne doivent pas être accolées au texte ou perdues dans le texte mais au contraire créer une indentation du texte.

- Utiliser au maximum 2 niveaux de puces pour déve lopper un point en sous-points.

Correspondance: Tristan Bourcier - Service du Professeur Laurent Laroche- CH N 0 des Q uinzeVingts 28 rue de Charenton - 75012 Paris- France - Tel : (33) 140021508 - Fax : (33) 140021599 mailto: bourcier@quinze-vingtsfr 


\section{C aractères}

- Les caractères doivent être assez grands pour être lus du fond dela salle de présentation. La taille idéale des polices de caractères est de 48-72 pour les titres et 28-40 pour le texte.

- Ne pas utiliser plus de 2 polices de caractère par pré sentation. Utiliser une police " sérif » (time new roman par exemple) pour lestitres et une police «sans sérif » (tahoma ou arial) pour le texte.

- Le meilleur moyen d'accentuation des caractères est l'utilisation de caractères gras ou de couleurs diffé rentes.

- Le soul ignage entraîne cependant une perte de la lisibilité car il entrâne une gêne à la lecture de la partie basse des lettres.

- Les majuscules utilisent beaucoup de place de par leur forme rectangulaire et imposent de disposer d'un double interligne dans le texte. Plus de 7 mots consécutifs en majuscules nécessitent d'être relus.

- La lisibilité des italiques varie d'une police à l'autre mais tend à être moins bonne que le style normal.

\section{Couleurs et fonds d'écran}

- Garder la même couleur de fond tout au long de la présentation.

- Ne pas utiliser plus de 4 couleurs par diapositive incluant celle de l'arrière plan.
- L'utilisation de caractères sombres sur fond d'écran clair (contraste positif), ou des caractères clairs sur fond d'écran sombre (contraste négatif) constitue le meilleur choix. Les caractères jaunes ou blancs sur fonds bleus, ou les caractères jaunes ou blancs sur fonds noirs ou verts réalisent les meilleurs contrastes négatifs. Le choix des couleurs doit pouvoir s'adapter en positif et négatif sel on l'intensité de l'éclairage du lieu de présentation.

- Ne pas utiliser de caractères rouges ou verts difficiles à percevoir en cas d'anomalies de la vision des couleurs.

\section{Graphiques, photographies, séquences vidéo}

- Les photographies peuvent être insérées tout au long de la présentation.

- Eviter la « surcharge » de données quantitatives dans les graphiques.

- D es séquences de film vidéo ajoutent encore plus de vécu et de force à la présentation.

\section{Références}

- Johns M . D esign for sides J Audiov M edia M ed. $1995 ; 18: 121-8$.

- Bellamy K. D esign standards for computer-generated teaching sides J Audiov M edia M ed. 1995; 18 : $115-20$.

- Bourcier T, Laroche L. Comment communiquer

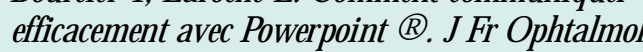
2005. Sous presse. 